\title{
Blut und Blutprodukte
}

\section{Blutgruppen}

\section{AB0-System}

- die Blutgruppe richtet sich nach der Antigeneigenschaft der Erythrozyten

- die Blutgruppenantigene A und B des AB0-Systems befinden sich an der Erythrozytenoberfläche. Das Antigen 0 gibt es nicht, man spricht allenfalls vom Merkmal $\mathrm{H}$

- die Blutgruppe A lässt sich in $A_{1}$ und $A_{2}$ unterteilen. Der Hauptunterschied zwischen den Untergruppen besteht darin, dass die Agglutination von $\mathrm{A}_{1}$-Erythrozyten bei Kontakt mit Anti-A-Serum wesentlich stärker und rascher verläuft. Für die Transfusion ist diese Unterteilung nicht von Bedeutung, da Antigen-Antikörper-Reaktionen zwischen $A_{1}$ und $A_{2}$ sehr selten auftreten und nur sehr schwach sind (Verteilung: $A_{1} \approx 20 \%, A_{2} \approx 80 \%$ )

\section{Rhesusfaktor}

- der Rhesusfaktor der Erythrozyten wird durch mehrere Antigene (Partialantigene) bestimmt (C, c, D, d, E, e)

- das Rhesusantigen $\mathbf{D}$ ist wegen seiner starken Immunität das wichtigste und bei Transfusionen stets zu berücksichtigen

- Blut, das Erythrozyten mit dem Antigen D besitzt, wird als Rhesus-positiv (Rh-pos) bezeich- net. Fehlt dieses Antigen, wird es als Rhesusnegativ (Rh-neg) bezeichnet

- Rhesusformel Ccddee (als Empfänger Rh-neg, als Spender Rh-pos)

\section{Weitere Blutgruppenantigene}

- Antigene: Kell, Duffy, Lewis, Kidd, Lutheran, P und MNSs

- Antikörper gegen diese Antigene werden erst nach Sensibilisierung gebildet

- Patienten, die Antikörper eines dieser Systeme besitzen, dürfen kein Blut mit dem entsprechenden Antigen erhalten

\section{Serumantikörper}

Antikörper sind Immunoglobuline und werden in reguläre und irreguläre Antikörper unterteilt.

\section{Reguläre Antikörper (Iso-Antikörper)}

- kommen regelmäßig im $\mathrm{AB} 0$-System, d. h. ohne Sensibilisierung vor (z. B. Anti-A, Anti-B). Sie werden jedoch erst im Lauf des ersten Lebensjahres entwickelt, d. h. Neugeborene besitzen in der Regel noch keine Iso-Antikörper des AB0Systems

- gehören zu der Klasse der IgM-Antikörper und sind wegen ihrer Größe nicht plazentagängig

- sie sind fast immer komplementbindend und somit hämolytisch wirksam 


\section{Irreguläre Antikörper}

- entstehen erst nach Sensibilisierung (z. B. nach vorangegangener Transfusion oder nach Schwangerschaft gebildete Antikörper)

- gehören zu der Klasse der IgM- oder IgG-Antikörper

- können gegen Untergruppen im AB0-System $\left(A_{2}, H\right)$ oder anderen Systemen (Rhesus, Kell, Duffy, Lewis, ...) gerichtet sein

- wichtig sind irreguläre Antikörper der IgGKlasse. Sie bleiben jahrelang nach Sensibilisierung erhalten und können eine lebensbedrohliche Transfusionsreaktion auslösen, außerdem sind sie plazentagängig, z. B. Rhesus

(Anti-D, Anti-C, ...), Kell (Anti-K), Duffy (Anti$\mathrm{Fy}^{\mathrm{a}}$ ), Lewis (Anti-Le ${ }^{\mathrm{a}}$ Anti-Le ${ }^{\mathrm{b}}$ )

- irreguläre AK gegen die Untergruppen im $\mathrm{AB} 0$ System (Anti- $\mathrm{A}_{1}$, Anti-H) besitzen sehr selten hämolytische Eigenschaften und sind somit klinisch nicht bedeutsam

- irreguläre Antikörper der IgM-Klasse sind z. B. Kälteagglutinine. Sie sind außer bei tiefer Hypothermie (z. B. in der Kardiochirurgie) ohne klinische Bedeutung, da ihr Temperaturoptimum bei $\approx 20^{\circ} \mathrm{C}$ liegt

\section{Blutgruppenhäufigkeiten}

\begin{tabular}{|l||c|}
\hline Blutgruppe & Häufigkeit (in Westeuropa) \\
\hline A & $43 \%$ \\
0 & $40 \%$ \\
B & $12 \%$ \\
AB & $5 \%$ \\
\hline Rh-positiv & $85 \%$ \\
Rh-negativ & $15 \%$ \\
\hline
\end{tabular}

\section{Blutprodukte}

\section{Frisches Vollblut (Frischblut)}

- weniger als $72 \mathrm{~h}$ altes Konservenblut (bis zu $6 \mathrm{~h}$ als Warmblut bezeichnet)

- Herstellung: frisches Vollblut einer Einzelspende wird mit 63-70 $\mathrm{ml}$ eines sterilen, pyrogenfreien Stabilisators (CPDA-1) in einem geschlossenen Blutbeutel gemischt
- Volumen: 450-570 ml

- Hämatokrit: $35-38,5 \%$ (abhängig vom Spender)

- Leukozyten $\approx 100 \%$, Plasma $\approx 100 \%$ (vom Vollblut)

- plasmatisches Gerinnungssystem und Thrombozyten nahezu vollständig erhalten. Die Thrombozyten sind jedoch nach spätestens $72 \mathrm{~h}$ nicht mehr funktionsfähig. Ebenso besteht rasch ein Defizit an Faktor V und VIII, da sie am lagerunginstabilsten sind

- höchste Immunität!

- hohe Infektionsgefahr (HIV, HBV, HCV, Lues $\rightarrow$ bei Lagerung der Blutkonserve $>72 \mathrm{~h}$ und $4{ }^{\circ} \mathrm{C}$ sterben Treponemen ab)

- darf nicht mehr in den Verkehr gebracht und transfundiert werden

\section{Vollblut}

- länger als $72 \mathrm{~h}$ gelagertes Frischblut (max. 21 Tage)

- Volumen: 450-570 ml

- Hämatokrit: 35-38,5\% (abhängig vom Spender)

- Leukozyten $\approx 100 \%$, Plasma $\approx 100 \%$ (vom Vollblut)

- nicht mehr funktionsfähige Thrombozytenreste

- Gerinnungsaktivität von Faktor V und VIII weitgehend aufgehoben (nach 10 Tagen noch $\approx 35 \%$, nach 20 Tagen $\approx 25 \%$ Aktivität)

- Mikrofilter $(10-40 \mu \mathrm{m})$ wegen Mikroaggregaten notwendig

- darf nicht mehr in den Verkehr gebracht und transfundiert werden

\section{Stabilisatoren und Additivlösungen für Erythrozytenkonzentrate}

Stabilisatoren dienen der Antikoagulation und Membranstabilität von Erythrozyten zur Lagerung.

\section{ACD-Stabilisator}

- Aqua destillata, Citrat (Acidum citricum, Natrium citricum), Dextrose

- Lagerung bei $2-6{ }^{\circ} \mathrm{C}$ (erschütterungsfrei) bis 21 Tage 


\section{CPD-A-1-Stabilisator}

- Citrat, Natriumdihydrogen-Phosphat, Dextrose, Adenin

- Lagerung bei $2-6{ }^{\circ} \mathrm{C}$ (erschütterungsfrei) bis 35 Tage

Additive Lösungen dienen der Aufrechterhaltung des Energiehaushalts und der Membranstabilität von Erythrozyten während der Lagerung und verlängern die Verwendbarkeit um 10-14 Tage gegenüber Stabilisatoren.

\section{SAG-M-Additivlösung}

- Sodiumchlorid ( $\mathrm{NaCl})$, Adenin, Glukose, Aqua ad inject., Mannitol

- Lagerung bei $2-6{ }^{\circ} \mathrm{C}$ (erschütterungsfrei) bis 42 Tage

\section{PAGGS-M-Additivlösung}

- Natrium-mono- und -di-hydrogen-Phosphat, Adenin, Glukose, Guanosin, Sodiumchlorid $(\mathrm{NaCl})$, Aqua ad inject., Mannitol

- Lagerung bei $2-6{ }^{\circ} \mathrm{C}$ (erschütterungsfrei) bis 49 Tage

Citrat $\rightarrow$ Antikoagulation (fällt ionisiertes Kalzium aus und hemmt somit Gerinnung)

Phosphat $\rightarrow$ Unterstützung der ErythrozytenGlykolyse; hebt $\mathrm{pH}$ leicht an $\rightarrow$ mehr 2,3-Diphosphoglycerat bleibt erhalten (bis zu 1 Woche 2,3-DPG normal) 2,3-DPG $\downarrow \Rightarrow$ Linksverschiebung der $\mathrm{O}_{2}$-Bindungskurve

$\Rightarrow$ schlechtere $\mathrm{O}_{2}$-Abgabe ans Gewebe (analog: $\mathrm{pH} \uparrow, \mathrm{CO}_{2} \downarrow$, Temp. $\downarrow$ )

Adenin $\rightarrow$ Lagerungsfähigkeitsverlängerung Dextrose, $\rightarrow$ Erythrozyten-Glykolyse $\rightarrow$ die enerGlukose giereichen Phosphate bleiben erhalten

\section{Lagerung}

- Frischblut, Vollblut und EK müssen bei $2-6{ }^{\circ} \mathrm{C}$ in geeigneten Kühlschränken oder -räumen mit fortlaufender Temperaturregistrierung gelagert werden. Die Kühlkette soll auch während des Transports nicht unterbrochen werden, sofern sie nicht unmittelbar danach verwendet werden

\section{Erythrozytenkonzentrat (EK)}

- alle verfügbaren EK enthalten in Abhängigkeit vom Herstellungsverfahren den größten Teil der Erythrozyten einer Vollbluteinheit

- sie unterscheiden sich im wesentlichen durch den Gehalt an noch verbleibenden Leukozyten und Thrombozyten („buffy coat"), Plasma (incl. Gerinnungsfaktoren) und Zusatz additiver Lösung zur Haltbarkeitsverlängerung

- $680 \mu \mathrm{g}$ Ammoniak pro EK!

- seit 2001 dürfen nur noch Leukozyten-depletierte zelluläre Blutkomponenten in den Verkehr gebracht werden

\section{Buffy-coat-haltiges EK}

- Herstellung: nach Zentrifugation des Vollblutes wird das Plasma durch einfache physikalische Verfahren im geschlossenen System teilweise oder weitgehend von den Erythrozyten getrennt

- Volumen: 280-320 ml (40-70 ml Plasma und $10 \mathrm{ml}$ Stabilisator)

- Hämatokrit: $>80 \%$

- Leukozyten: $\approx 90 \%$, Plasma: 20-30\% (vom Vollblut)

\section{Buffy-coat-freies EK}

- Herstellung: nach Zentrifugation des Vollblutes wird das Plasma und der Buffy-coat (Leukozyten und Thrombozyten) durch physikalische Verfahren im geschlossenen System teilweise oder weitgehend von den Erythrozyten getrennt. Zur Verbesserung der Konservierung wird das EK anschl. mit 40-70 ml Plasma resuspendiert

- Volumen: 250-300 ml (40-70 ml Plasma und 10 $\mathrm{ml}$ Stabilisator)

- Hämatokrit: $>80 \%$

- Leukozyten: < 50\%, Plasma 20-30\% (vom Vollblut)

\section{Buffy-coat-freies EK in additiver Lösung}

- Herstellung: das Buffy-Coat-freie EK wird in 80-100 ml Additivlösung aufgeschwemmt

- Volumen: 280-350 ml (10-25 ml Plasma) 
- Leukozyten: $<20 \%$, Plasma: < 15\% (vom Vollblut)

\section{Leukozyten-depletiertes EK (gefiltertes EK)}

- Herstellung: mittels spezieller Tiefenfilter (Leukozytendepletionsfilter) wird die Anzahl der Leukozyten weiter reduziert. Die Anzahl der Restleukozyten darf $1 \times 10^{6}$ Zellen pro EK nicht übersteigen. Leukozyten-depletierte EK können sowohl aus Buffy-Coat-freien EK als auch aus Buffy-Coat-freien EK in additiver Lösung hergestellt werden

- Nachteile: Kontaminationsgefahr und fehlende Lagerungsfähigkeit bei Eröffnung des geschlossenen Systems: Sie sollten nach Eröffnen möglichst umgehend verwendet werden

- Leukozyten: $<1 \%$, Plasma: $<20 \%$ (vom Vollblut)

Ind:

- Prävention einer Alloimmunisierung gegen leukothrombozytäre Merkmale bei absehbarer Langzeitsubstitution und Immunsuppression (auch vor Transplantation)

- hämatologische Grunderkrankungen (aplastische Anämie, myelodysplastische Syndrome, transfusionspflichtige chronische Anämien, Leukämien)

- Schwangere, wenn CMV-negative EK nicht verfügbar sind (Vermeidung einer intrauterinen fetalen CMV-Infektion) und ggf. HIV-Infizierte

- herzchirurgische Patienten mit einem Transfusionsbedarf > 3 EK (seit 1999 in England und der Schweiz praktiziert) $\rightarrow$ geringe Inzidenz an Infektionen und geringere postoperative Mortalität

- Zustand nach nichthämolytischer, febriler Transfusionsreaktion

- Verhinderung des Refraktärzustandes gegen Thrombozyten

- Reduzierung von intrazellulären, leukozytären Virenübertragung (CMV, HIV)

- Prophylaxe des ARDS bei Massivtransfusion

- evtl. Früh-, Neugeborene und Säuglinge bis zum ersten Lebensjahr

\section{Gewaschenes EK}

- Herstellung: durch mehrmaliges Aufschwemmen und Zentrifugieren leukozyten-depletierter Erythrozyten wird der größte Teil des Plasmas, der Leukozyten und Thrombozyten entfernt.

- Leukozyten: < $1 \%$, Plasma: < $1 \%$ (vom Vollblut)

- Nachteile: Kontaminationsgefahr und fehlende Lagerungsfähigkeit bei Eröffnung des geschlossenen Systems sowie waschbedingte Zellschäden

Ind:

- Unverträglichkeit gegen Plasmaproteine, trotz Verwendung von Leukozyten-depletierten EK in additiver Lösung oder bei Nachweis von Antikörpern gegen IgA oder andere Plasmaproteine

\section{Kryokonserviertes EK}

- Herstellung: gewaschene EK werden unter $\mathrm{Zu}$ satz eines Gefrierschutzmittels (Glycerin) tiefgefroren und bei mindestens $-80^{\circ} \mathrm{C}$ gelagert. Kryokonservierte EK sind praktisch frei von Plasma sowie intakten Leukozyten und Thrombozyten. Nach dem Auftauen muss das Glycerin wieder ausgewaschen und die EK müssen umgehend verwendet werden

- Leukozyten: < $1 \%$, Thrombozyten: $<1 \%$, Plasma: $<1 \%$ (vom Vollblut)

Ind:

- nur bei Patienten mit komplexen Antikörpergemischen oder mit Antikörpern gegen ubiquitäre Antigene, die nicht anders versorgt werden können

\section{Bestrahltes EK}

- Herstellung: Bestrahlung mit 30 Gy kurz vor der vorgesehenen Transfusion. Zerstörung immunkompetenter Lymphozyten. Nach Möglichkeit sollten leukozyten-arme gefilterte EK bestrahlt werden

- Nachteil: der lagerungsbedingte Kaliumaustritt aus den Erythrozyten wird durch Bestrahlung zusätzlich verstärkt 


\section{Absolute Indikation}

- intrauterine Transfusion

- Neugeborene $(<37$. SSW $)$

- Stammzell- oder Knochenmarktransplantation

- autologe Stammzellenentnahme

- lymphoproliferative Erkrankungen

- Immundefizit-Syndrom

- alle gerichteten Blutspenden aus der engen Familie

\section{Relative Indikation}

- Patienten mit Malignom unter Hochdosis-Chemotherapie

- Autoimmunerkrankungen

- Morbus Hodgkin

- Transplantation solider Organe (Immunsuppression)

- Austauschtransfusion

- für Kinder und Patienten vor/nach Transplantation sollten nur CMV-freie und bestrahlte Konserven verwendet werden!

- die Gabe von Fremdblut führt zu einer klinisch fassbaren Immunsuppression bei reduzierter „natural killer cell activity“ und reduzierter T-Zell-Entwicklung $\rightarrow$ verminderte AbstoBungsreaktion nach Nierentransplantation, günstige Beeinflussung des postoperativen Verlaufs von Autoimmunerkrankungen wie z. B. Morbus Crohn, jedoch erhöhte Tumorrezidivrate nach EK-Gabe von Karzinompatienten

\section{Fresh-frozen-Plasma (FFP)}

- Herstellung: innerhalb von $6 \mathrm{~h}$ (max. $24 \mathrm{~h}$ ) tiefgefrorenes Plasma, welches aus einer Vollblutspende $(\approx 270 \mathrm{ml}$ ) oder durch Plasmapharese $(\approx 600 \mathrm{ml})$ gewonnen worden ist

- Antikoagulanzien: Citrat-Phosphat-DextroseAdenin (CDPA)

- physiologische Zusammensetzung prokoagulatorischer und profibrinolytischer Faktoren

- Gerinnungsaktive Qualität von Frischplasmen abhängig von

- Konzentration beim Spender (große interindividuelle Schwankungen bei Spendern von o,6-1,4 U/ml jedes Gerinnungsfaktors, dabei entspricht $1 \mathrm{U} / \mathrm{ml}$ 100\% Aktivität eines Plasmapools)

- Lagerung (Temperatur)

- Herstellungsverfahren (Virusinaktivierung durch Methylenblau, Hitze,...)

- Auftauen (Temperatur und Geschwindigkeit): Soll: $25 \mathrm{~min}$ bei $37^{\circ} \mathrm{C}$

- Die Aktivität des Gerinnungsfaktors VIII im aufgetauten Plasma soll mind. $70 \%$ der individuellen Ausgangsaktivität sein (also mind. $0,7 \mathrm{U} / \mathrm{ml}$, von BGA vorgeschrieben).

- Nach dem Auftauen verlieren sie jedoch rasch an Aktivität $\approx 60-70 \%$ der Ausgangsaktivität nach dem Auftauen, außer Faktor $\mathrm{V}(\approx 40-50 \%)$, da sehr labil $\rightarrow$ FFP inner-

\section{Übersicht Erythrozytenkonzentrate}

\begin{tabular}{|l|l|l|l|l|l|}
\hline & \multicolumn{2}{|c|}{ Restanteil des Vollblutes (\%) } \\
\hline Präparat & $\begin{array}{l}\text { Volumen } \\
(\mathrm{ml})\end{array}$ & $\begin{array}{l}\text { Hämato- } \\
\text { krit (\%) }\end{array}$ & $\begin{array}{l}\text { Erythrozyten- } \\
\text { masse }\end{array}$ & $\begin{array}{l}\text { Leuko- } \\
\text { zyten }\end{array}$ \\
\hline Vollblut & $450-570$ & $35-38,5$ & 100 & 100 & 100 \\
\hline $\begin{array}{l}\text { Buffy-coat-haltiges EK } \\
\text { Buffy-coat-freies EK }\end{array}$ & $280-320$ & $60-80$ & $\approx 90$ & $\approx 90$ & $20-30$ \\
$\begin{array}{l}\text { Buffy-coat-freies EK } \\
\text { in additiver Lösung }\end{array}$ & $250-300$ & $60-80$ & $\approx 90$ & $<50$ & $20-30$ \\
\hline $\begin{array}{l}\text { Leukozyten-depletiertes EK } \\
\text { gewaschenes EK }\end{array}$ & $250-350$ & $50-70$ & $>80$ & $<20$ & $<15$ \\
kryokonserviertes EK & $200-350$ & $50-80$ & $>80$ & $<1$ & $<20$ \\
\end{tabular}


halb einer $1 / 2 \mathrm{~h}$ nach dem Auftauen geben! nach $4 \mathrm{~h}$ nur noch $40-50 \%$ Aktivität vorhanden, nach $6 \mathrm{~h} \% \%$

- zulässiger Restzellgehalt:

Erythrozyten: < 1000/ $\mu$ l, Leukozyten: $<500 / \mu \mathrm{l}$, Thrombozyten: $<20.000 / \mu 1$

- Proteinkonzentration: $60 \mathrm{~g} / \mathrm{l}$

- Lagerung: bei $-30{ }^{\circ} \mathrm{C}$ : bis $1 \mathrm{Jahr}$, bei $-40{ }^{\circ} \mathrm{C}$ : bis 2 Jahre, bei $-70^{\circ} \mathrm{C}$ : bis 3 Jahre

Ind:

- Verdünnungskoagulopathie infolge Massivtransfusion

- Verbrauchskoagulopathie

- Lebererkrankungen mit aktiver klinischer Blutung

- angeborener Faktor-V- und -XI-Mangel (es gibt keine Einzelfaktorenpräparate hierfür)

- Plasmaaustausch bei Moschkowitz-Syndrom, thrombotisch-thrombozytischer Purpura (wird zur Zeit als Therapie der Wahl angesehen)

- Guillain-Barré-Syndrom (der mehrfache Plasmaaustausch ist einer rein supportiven Therapie nachweislich überlegen)

- Austauschtransfusionen (von mehr als dem errechneten Blutvolumen des Patienten) bei Kindern und Erwachsenen

- Notfallindikation beim Hämophiliepatienten

- Gabe von FFP bei Kindern:

- bei Quick < 40\%, PTT > 150\% der Norm und Fibrinogen $<0,75 \mathrm{~g} / \mathrm{l}$ bzw.

- spätestens bei 1-1,5fachen Verlust des geschätzten Blutvolumens

\section{Dosis}

Faustregel: $1 \mathrm{ml} / \mathrm{kg}$ FFP $\Rightarrow$ Erhöhung des

Faktorengehalts um $\approx 1-2 \%$

Massivtranfusion: - EK: FFP $=3: 1$ bis notfalls $1: 1$

Leberausfall: $\quad-10-20 \mathrm{ml} / \mathrm{kg}$, initial 4 Einheiten, Tagesbedarf $\approx 8$ Einheiten

KI:

- Plasmaeiweißallergie

- Mangel einzelner Gerinnungsfaktoren

- Volumenmangel ohne Gerinnungsstörungen

- Hypervolämie, Hyperhydratation, Lungenödem
NW:

- Überempfindlichkeitsreaktionen

- Herz-Kreislauf-Reaktionen infolge von Citratreaktionen bei Leberfunktionsstörungen, sowie bei Neugeborenen, bes. bei schneller Transfusion

- Immunisierung des Empfängers gegen Plasmaproteine

- transfusionsinduzierte akute Lungeninsuffizienz (TRALI-Syndrom): sehr selten und tritt fast ausschließlich durch Übertragung größerer Mengen Plasma, das granulozytenspezifische Antikörper enthält, auf

- mit nichtinaktiviertem Plasma können Erreger von Infektionskrankheiten (z. B. HBV, HCV, CMV, HIV, Parvovirus B19) oder andere Mikroorganismen übertragen werden

- Virusinaktivierung des Plasmas durch

- Hitzebehandlung

- Alkoholfraktionierung

- photodynamische Einzelplasmabehandlung mit Methylenblau und Lichtexposition

- Behandlung von Poolplasma mit Solvent/Detergent-Verfahren (S/D): Tri-N-butylphosphat $\rightarrow$ hoher Verlust der Aktivität von Faktor V und VIII

- seit 01.07.1995: Lagerung von 4 Monaten vorgeschrieben $\rightarrow$ Quarantäneplasma

\section{Blutgruppenkompatible Gabe von FFP}

\begin{tabular}{|l|l|}
\hline Patient (Empfänger) & Kompatible FFP \\
\hline A & A (AB) \\
B & B (AB) \\
AB & AB \\
0 & $0(A, B, A B)$ \\
\hline
\end{tabular}

- Plasma der Blutgruppe AB kann im Notfall für Patienten aller Blutgruppen verwendet werden

- das Rhesus-System braucht nicht berücksichtigt zu werden

\section{Thrombozytenkonzentrat (TK)}

Herstellung:

- Leukozytendepletiertes Pool-Thrombozytenkonzentrat: im geschlossenen System werden in 
der Regel 4-6 AB0-blutgruppengleiche „buffy coats" oder fertige Einzelspenderthrombozytenkonzentrate zusammengeführt und durch anschließende Filtration das leukozytendepletierte Pool-Thrombozytenkonzentrat hergestellt (enthält in Abhängigkeit von der Anzahl gepoolter Einheiten $240-360 \times 10^{9}$ Thrombozyten in 200-350 ml Plasma oder einer Plasmaersatzlösung

- Leukozytendepletiertes Apherese-Thrombozytenkonzentrat: die Herstellung erfolgt durch Apherese, entweder durch Thrombozytapherese oder Multikomponentenspende, mit anschließender Leukozytendepletion oder durch ein Aphereseverfahren mit integrierter Leukozytendepletion (enthält in der Regel $200-400 \times 10^{9}$ Thrombozyten in etwa 200-300 ml Plasma eines Einzelspenders)

- im TK ist eine geringe Menge von Erythrozyten $\left(<3 \times 10^{9}\right)$ vorhanden. Der Gehalt an Restleukozyten liegt unterhalb von $1 \times 10^{6}$ pro TK

\section{Lagerung:}

- unter ständiger Bewegung (auf Rüttelmaschine) bei Raumtemperatur $\left(>22 \pm 2{ }^{\circ} \mathrm{C}\right)$ für max. $3-5$ Tage haltbar (nicht im Kühlschrank, dies führt zur Plättchenaggration!).

\section{Ind:}

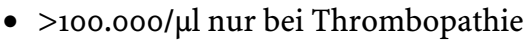

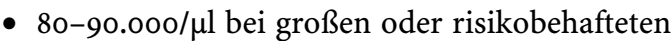
Operationen (bes. Kardiochirurgie, Neurochirurgie, Augen)

- 50-60.000/ $\mu \mathrm{l}$ bei Massivtransfusion

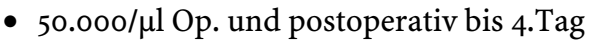

- 20-50.000/ $\mu$ l bei Blutung

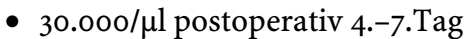

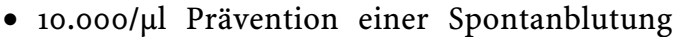
ohne chirurgischen Eingriff (nach LTPL evtl. erst bei $<10.000 / \mu$ l wegen möglicher Sensibilisierung)

- Cave: nicht bei Pseudothrombopenien (fälschlich zu niedrig gemessene Werte durch antikörperinduzierte Verklumpung, z. B. EDTAabhängige Thrombopenie $\rightarrow$ Bestimmung im Citratblut)

\section{Dosis}

Faustregel: minimaler Thrombozytenbedarf: Thrombozytenanzahl = gewünschter Thrombozytenanstieg $(/ \mu \mathrm{l}) \times$ Blutvolumen $(\mathrm{ml})(\approx 70 \mathrm{ml} /$ $\mathrm{kg}) \times 1,5$

z. B. Anstieg um $50.000 / \mu \mathrm{l}$, Patient $70 \mathrm{~kg}$ :

- $50 \times 10^{3} / \mu \mathrm{l} \times 70 \mathrm{~kg} \times 70 \mathrm{ml} / \mathrm{kg} \times 1,5=$

- $50 \times 10^{3} / \mu \mathrm{l} \times 4900 \times 10^{3} \mu \mathrm{l} \times 1,5=$

- $367 \times 10^{9} \approx 3,6 \times 10^{11}$

Erfahrungsgemäß führt

- 1 Einheit Pool-TK oder

- 1 Einheit Apherese-TK

zu einem Thrombozytenanstieg von ca. 20.000$30.000 / \mu \mathrm{l}$

nur $60-70 \%$ finden sich in der Blutzirkulation wieder, der Rest wird bei Erstpassage in der Milz abgefangen (daher $\times 1,5)$

- seit 2001 dürfen nur noch Leukozyten-depletierte zelluläre Blutkomponenten in den Verkehr gebracht werden

\section{Blutgruppenkompatible Transfusion von TK}

\begin{tabular}{|l|l|}
\hline Patient (Empfänger) & Kompatible TK \\
\hline A & A (0) \\
B & B $(0)$ \\
AB & AB $(\mathrm{A}, \mathrm{B}, 0)$ \\
0 & 0 \\
\hline Rh-positiv & Rh-positiv (Rh-negativ) \\
Rh-negativ & Rh-negativ (evtl. Rh-positiv) \\
\hline
\end{tabular}

- Übertragung nach Kompatibilität im AB0- und Rh-System wie bei EK, wegen der geringen, aber immer vorhandenen Kontamination mit Erythrozyten. Weitere wichtige Alloantigene sind die HLA-Antigene der Klasse I sowie plättchenspezifische Antigene

- einem Rh-neg-Empfänger dürfen Rh-posThrombozyten nur im Notfall transfundiert werden, da der Empfänger Antikörper bildet, die oft lebenslang erhalten bleiben. Wird einem solchen Patienten erneut Rh-pos-Blut übertragen, kann eine schwere hämolytische Transfusionsreaktion ausgelöst werden. Wenn die Gabe von Rh-pos-Thrombozyten unvermeidlich ist, sollte bei Rh-neg-Frauen im gebärfähigen Alter 
eine Prophylaxe mit Anti-D-Immunoglobulin (250-300 $\mu$ g Anti-D i.v.) durchgeführt werden (Cave: keine i.m.-Injektion)

- Gabe über ein spezielles Thrombozytenbesteck (Filter 170-200 $\mu \mathrm{m}$ ), das einen geringeren Thrombozytenverlust im System verursacht

- Therapiekontrolle: Thrombozytenzahl und Thrombozytenfunktion

- bei immunsupprimierten Patienten muss vor TK-Transfusion zur Vermeidung einer GvHReaktion eine Bestrahlung mit ca. 30 Gy durchgeführt werden

\section{Transfusion}

\section{Indikationen zur Transfusion}

- für die Indikation zur Transfusion von EK's lassen sich keine obligaten unteren Grenzwerte für Hämoglobin oder Hämatokrit festlegen

- nach neueren Empfehlungen wird bei bestehenden kardialen Kompensationsmechanismen die minimale $\mathrm{Hb}$-Konzentration bei $6,0 \mathrm{~g} / \mathrm{dl}$ angegeben! (= kritischer Hb-Wert, bei dem bei Normovolämie und Normoxie die $\mathrm{O}_{2}$-Versorgung des Gewebes noch gewährleistet ist)

Aktuelle Indikationen zur Transfusion sind:

1. eine Hb-Konzentration $<\mathbf{6 , 0}$ g/dl bzw. HKt $<$ $20 \%$

2. eine $\mathrm{Hb}$-Konzentration zwischen $6, \mathrm{o}$ und $10, \mathrm{og} /$ dl und bei

- $\mathrm{p}_{\mathrm{v}} \mathrm{O}_{2}<32 \mathrm{mmHg}$
- $\mathrm{O}_{2}$-Extraktionsrate $>50 \%$

- ein um mehr als 50\% von der Ausgangssituation gesunkener $\mathrm{O}_{2}$-Verbrauch, der nicht anderweitig geklärt werden kann

- myokardiale und zerebrale Ischämieanzeichen trotz ausreichender Isovolämie $\rightarrow$ STStreckensenkungen $>0,1 \mathrm{mV}$ oder ST-Hebungen $>0,2 \mathrm{mV}$ für eine Dauer von mind. 1 min in den Ableitungen II und $\mathrm{V}_{5}$

- die restriktive Gabe von Erythrozytenkonzentraten (Hb-Transfusionswert $<7,0 \mathrm{~g} / \mathrm{dl}$ vs. $<10 \mathrm{~g} / \mathrm{dl}$ ) führte in einer von Herbert veröffentlicht großen randomisierten Studie zu keiner Zunahme der 30-Tage- und der Krankenhausmortalität.

Bei Fieber und SIRS/Sepsis können individuell Hb-Konzentrationen $>10 \mathrm{~g} / \mathrm{dl}$ sinnvoll sein!

Mehr als 15 Tage lang gelagerte Erythrozytenkonzentrate scheinen ungeeignet zu sein, die globale und lokale $\mathrm{O}_{2}$-Versorgung beim kritischkranken Patienten zu verbessern!

\section{Maximal tolerabler Blutverlust (MTBV)}

$$
\text { MTBV }=\frac{\begin{array}{c}
\text { geschätztes Blutvolumen } \\
(70 \mathrm{ml} / \mathrm{kg}) \times\left(\mathrm{Hkt}_{\mathrm{o}}-H \mathrm{Ht}_{\mathrm{min}}\right)
\end{array}}{\left(\mathrm{Hkt}_{\mathrm{o}}-\mathrm{Hkt}_{\mathrm{min}}\right) / 2}
$$

$\mathrm{Hkt}_{\mathrm{o}}=$ Ausgangshämatokrit, $\mathrm{Hkt}_{\min }=$ minimaler Hämatokrit

- für das Überleben von (Myokard)Gewebe ist ein unterer $\mathrm{O}_{2}$-Gehalt von $6 \mathrm{ml} / \mathrm{dl}$, was einem $\mathrm{Hb}$ Wert von 4,4 g/dl unter Raumluft entspricht, notwendig. Es liegen einzelne Berichte vor, dass Zeugen-Jehovas-Patienten Hb-Werte von 2,4 g/dl

\begin{tabular}{|c|c|c|c|c|}
\hline \multirow[b]{2}{*}{ Alter } & \multicolumn{2}{|c|}{ Transfusionsgrenzen } & \multicolumn{2}{|c|}{ Normalwerte } \\
\hline & $\mathrm{Hb}(\mathrm{g} / \mathrm{dl})$ & Hk (\%) & $\mathrm{Hb}(\mathrm{g} / \mathrm{dl})$ & Hk (\%) \\
\hline Frühgeborene & $12-14$ & $40-50$ & & \\
\hline Frühgeborene bis 2 Monate & $11-12$ & $36-42$ & & \\
\hline Neugeborene & 10 & $30-40$ & $15-25$ & $45-65$ \\
\hline Säuglinge in der Trimenonreduktion & 8 & $25-28$ & $9-12$ & $30-42$ \\
\hline 1 Jahr & $6-7$ & $20-25$ & $10-15$ & $35-45$ \\
\hline 6 Jahre & $6-7$ & $20-25$ & $10-15$ & $35-45$ \\
\hline Gesunder Erwachsener & $6-7$ & 20 & $12-16$ & $40-50$ \\
\hline KHK-Patient & 10 & 30 & & \\
\hline
\end{tabular}

\section{Hb-Hk-Normalwerte und kritische Grenzwerte}


und Hkt-Werte von bis zu $4 \%$ ohne Organschäden überlebten $\rightarrow$ das Recht auf Selbstbestimmung (Art. 2 GG) ist bei erwachsenen bewusstseinsklaren Patienten zu respektieren (gegenüber dem Grundsatz der ärztlichen Behandlungsfreiheit). Anders hingegen bei minderjährigen Kindern, deren Eltern eine Bluttransfusion verweigern. Hier muss über das Vormundschaftsgericht eine Einwilligung zur Transfusion gegen den Willen der Eltern eingeholt werden $(\$ 1666$ BGB). Im Notfall muss die Transfusion erfolgen, da sonst der Tatbestand der unterlassenen Hilfeleistung zugrunde liegen kann

\section{Therapievorschlag}

\begin{tabular}{|l|l|}
\hline Volumenverlust & Therapie \\
\hline $\begin{array}{l}\text { Blutverlust bis 20\% } \\
\text { des Blutvolumens } \\
\text { Blutverlust ab 30\% } \\
\text { des Blutvolumens }\end{array}$ & $\begin{array}{l}\text { Ersatz mit Kristalloiden und } \\
\text { Kolloiden } \\
\text { EK-Einsatz nach Hb-Wert } \\
\text { FFP-Gabe im Verhältnis 4:1-2:1 } \\
\text { (EK:FFP) }\end{array}$ \\
$\begin{array}{l}\text { ab Verlust des ein- } \\
\text { fachen Blutvolumens }\end{array}$ & $\begin{array}{l}\text { FFP-Einsatz nach Hb-Wert } \\
\text { (EK:FFP) }\end{array}$ \\
& $\begin{array}{l}\text { EK-Einsatz nach Hb-Wert } \\
\text { ab Verlust des 1,5- } \\
\text { fachen Blutvolumens }\end{array}$ \\
& $\begin{array}{l}\text { FFP-Gabe im Verhältnis 1:1 } \\
\text { (EK:FFP) }\end{array}$ \\
& $\begin{array}{l}\text { TK-Gabe im Verhältnis 1:1 (EK:TK) } \\
\text { bzW. ab 50.000 Thrombozyten/ } \mu l\end{array}$ \\
&
\end{tabular}

Unter extremer Hämodilution sind Gelatinelösungen aufgrund eines erhöhten Transportvermögens von $\mathrm{CO}_{2}$ und keiner über das $\mathrm{Maß}$ des Hämodilutionseffektes hinausgehende Beeinflussung der Gerinnung zu bevorzugen

\section{Dosis}

Faustregel: $3-4 \mathrm{ml} / \mathrm{kg} \mathrm{EK} \Rightarrow$ Erhöhung des $\mathrm{Hb}$ $\mathrm{um} \approx 1 \mathrm{~g} / \mathrm{dl}$

oder:

erforderl. Vol. $=$

$\frac{\text { Blutvolumen }(\approx 70 \mathrm{ml} / \mathrm{kg}) \times \mathrm{Hkt}_{\text {wunsch }}-\mathrm{Hkt}_{\text {Aktuell }}}{H \mathrm{kt} \mathrm{t}_{\text {tranfBlut }}}$

$\mathrm{Hkt}_{\text {Wunsch }}=$ gewünschter Hämatokrit

$\mathrm{Hkt}_{\text {Aktuell }}=$ aktueller Hämatokrit

$\mathrm{Hkt}_{\text {tranf.Blut }}=$ Hämatokrit der transfundierten Konserve (60-80\%)

\section{Verträglichkeitstests (Prophylaxe hämolytischer Transfusionsreaktionen)}

Vor jeder Transfusion müssen folgende Untersuchungen bzw. Tests durchgeführt werden:

- Bestimmung der Blutgruppe und des Rh-Faktors

- Antikörpersuchtest (indirekter Coombs-Test) beim Empfänger und Spender

- Kreuzprobe

- Überprüfung des Blutgruppenbefundes, der Kreuzprobe und der Konserve

- Bedsidetest

\section{Bestimmung der Blutgruppe und des Rh-Faktors}

\section{Bestimmung der Blutgruppe}

\begin{tabular}{l|ll|ll}
$\begin{array}{l}\text { Blut- } \\
\text { gruppe }\end{array}$ & $\begin{array}{l}\text { Erythrozytenreak- } \\
\text { tion mit Testserum } \\
\text { (Bedsidetest) }\end{array}$ & $\begin{array}{l}\text { Serumreaktion } \\
\text { mit Testerythro- } \\
\text { zyten }\end{array}$ \\
\hline & Anti-A & Anti-B & A-Zellen & B-Zellen \\
\hline A & + & - & - & + \\
B & - & + & + & - \\
AB & + & + & - & - \\
0 & - & - & + & + \\
\hline
\end{tabular}

\section{Kreuzprobe}

Mit der Kreuzprobe soll festgestellt werden, ob sich Antikörper beim Spender oder Empfänger befinden und eine hämolytische Transfusionsreaktion auslösen können. Die Kreuzprobe besteht aus 3 Stufen:

Stufe 1 = Kochsalztest (= eigentliche Kreuzprobe)

- Die Erythrozyten des Spenders werden mit dem Serum des Empfängers (Majorteil) und umgekehrt (Minorteil) zusammengebracht.

\section{Majortest}

- Das Empfängerserum wird auf Antikörper gegen Spendererythrozyten untersucht.

\section{Minortest}

- Spenderserum wird auf Antikörper gegen Empfängererythrozyten untersucht 
- bes. wichtig bei Neugeborenen und Kleinkindern mit noch nicht ausgereiftem Immunsystem

- tritt beim Major- oder Minortest nach Inkubation von 5 min bei Raumtemperatur und anschließender Zentrifugation schon eine Agglutination auf, besteht Unverträglichkeit und die weiteren Tests können weggelassen werden

\section{Stufe 2 = Albumintest}

- Suche nach kompletten Antikörpern oder Antikörpern, die in Kochsalz keine Agglutination hervorrufen

- Zugabe von 30\%-igem Rinderalbumin und Inkubation von $30-45$ min bei $37^{\circ} \mathrm{C}$

- nach Zentrifugation wird auf Agglutination untersucht

\section{Stufe $\mathbf{3}$ = Coombs-Test (direkter Coombs-Test)}

- Die Suche nach inkompletten Antikörpern, die erst durch Zugabe von Coombs-Serum (Antihumanglobulin) eine sichtbare Agglutination bewirken. Die im Coombs-Serum enthaltenen Antikörper bilden eine „Verbindungsbrücke“ zwischen inkompletten Antikörpern.

\section{Antikörpersuchtest (indirekter Coombs-Test)}

Bei Empfänger und Spender

- hier werden im Unterschied zur Kreuzprobe gepoolte Testerythrozyten mit einer optimalen Anzahl von Antigenen mit Empfänger- bzw. Spenderserum vermischt

- Aufdeckung der meisten irregulären bzw. inkompletten Antikörper wie z.B. Rhesus, Kell, Duffy, Lewis, Kidd, ...

- eine weitere Identifizierung von irregulären Antikörpern erfolgt dann gegebenenfalls mit speziellen Testerythrozyten

\section{Bedsidetest}

- mit dem Bedsidetest sollen Vertauschungen und Verwechslungen bei der Blutabnahme, bei der Kreuzprobe oder bei der Zuordnung der Blutpräparate zum Patienten entdeckt werden

- der Bedsidetest ist unmittelbar vor der Transfusion vom transfundierenden Arzt oder unter seiner Aufsicht durchzuführen, um die AB0-Blutgruppe des Empfängers zu bestätigen. Das Ergebnis ist schriftlich zu dokumentieren. Eine Testung der Konserve ist nicht mehr vorgeschrieben!

- eine Bestimmung des Rhesusfaktors oder eine Blutgruppenkontrolle des EK („Inhaltskontrolle") ist nicht vorgeschrieben

- bei Eigenblut muss der Bedsidetest vom Empfänger und von der Eigenblutkonserve („Inhaltskontrolle") durchgeführt werden, um Vertauschungen zu vermeiden, da hier keine Kreuzprobe erfolgt

\section{(D) Maßnahmen vor Transfusion}

Vor Beginn der Transfusion hat der transfundierende Arzt persönlich zu überprüfen:

- den Blutgruppenbefund des Empfängers und evtl. vorliegende irreguläre Antikörper

- ob die Konserve für den entsprechenden Empfänger bestimmt ist

- ob die Blutgruppe der Konserve (Konservenetikett) dem Blutgruppenbefund des Empfängers entspricht

- ob Verträglichkeit besteht (negative Kreuzprobe) und die Kreuzprobe noch Gültigkeit besitzt (in der Regel $72 \mathrm{~h}$ )

- ob die angegebene Konservennummer mit dem Begleitschein übereinstimmt

- ob die Konserve unversehrt und das Verfallsdatum nicht überschritten ist

- Durchführung des Bedsidetests (oder unter seiner Aufsicht)

\section{Auswahl von Erythrozyten- konzentraten}

\section{Blutgruppenkompatible Transfusion von EK}

\begin{tabular}{|l|l|}
\hline Patient (Empfänger) & Kompatible EK \\
\hline A & A (0) \\
B & B $(0)$ \\
AB & AB $(A, B, 0)$ \\
0 & 0 \\
Rh-positiv & Rh-positiv (Rh-negativ) \\
Rh-negativ & Rh-negativ (evtl. Rh-positiv) \\
\hline
\end{tabular}

nach Möglichkeit sollte AB0- und Rh-blutgruppengleich transfundiert werden 
- einem Rh-neg-Empfänger darf Rh-pos-Blut nur im Notfall transfundiert werden, da der Empfänger Antikörper bildet, die oft lebenslang erhalten bleiben. Wird einem solchen Patienten erneut Rh-pos-Blut übertragen, kann eine schwere hämolytische Transfusionsreaktion ausgelöst werden

- die Gabe von Rh-positivem EK sollte bei Rh-negKindern und Rh-neg-Frauen im gebärfähigen Alter unbedingt vermieden werden

- „Universalspenderblut 0 “

Erythrozyten der Blutgruppe 0 lassen sich praktisch reaktionslos auf blutgruppenungleiche Empfänger übertragen. Da jedoch in EK der Blutgruppe 0 immer noch ein Plasmaanteil mit Anti-A- und Anti-B-Antikörpern vorhanden ist, ist die Menge der übertragbaren EK begrenzt. Bei größeren Transfusionsmengen werden die Empfängererythrozyten geschädigt, da dann die Verdünnung der Antikörper nicht mehr ausreichend hoch ist.

Bei EK mit geringem Plasmaanteil (gewaschene EK) brauchen die Isoantikörper des AB0-Systems im Spenderplasma nicht berücksichtigt werden. Solche EK können im Bedarfsfall unter Berücksichtigung der Majorkompatibilität im AB0-System unbedenklich übertragen werden

- bei Austauschtransfusionen an Neugeborenen muss das für den Austausch herangezogene EK mit der AB0-Blutgruppe der Mutter und des Kindes kompatibel sein

\section{Mikroaggregate und Blutfiltration}

- bei der Lagerung von Blutkonserven entstehen durch Alterung der Blutbestandteile Mikroaggregate, die sich durch Stabilisatoren- und Antikoagulanzienzusatz nicht verhindern lassen. Auchin Blutpräparaten mit neueren additiven Lösungen lassen sich Mikroaggregate nachweisen

- sie setzen sich zusammen aus gealterten, zerfallenen oder degenerierten Thrombozyten, Leukozyten, Zellfragmenten, Fibrin, Lipiden und denaturierten Proteinen

- bereits nach wenigen Stunden kommt es zur Thrombozytenaggregation, nach $24-48 \mathrm{~h} \mathrm{zu}$ stabilen Mikroaggregaten
Die Übertragung von Mikroaggregaten

- spielt eine wichtige Rolle bei der Entwicklung der Posttransfusionslunge

- führt zur Aktivierung körpereigener Thrombozyten mit Sequestration in der Milz $(\rightarrow$ Thrombozytopenie 2-4 Tage nach Transfusion)

\section{Blutfiltertypen}

Flächenfilter sind Siebe aus Polyester mit Poren einer definierten Größe von 10-200 $\mu \mathrm{m}$. Partikel, die größer als die jeweiligen Poren sind, werden mechanisch abgeschieden. Flächenfilter sind z. B. der Standardfilter $(170 \mu \mathrm{m})$, PALL-Ultipor SQ 40 $(40 \mu \mathrm{m})$, Mikrofilter MF $10(10 \mu \mathrm{m})$ oder Microtrans $(10 \mu \mathrm{m})$.

Kaskadenfilter bestehen aus 3-4 hintereinander geschalteten Flächenfiltern mit zunehmend kleineren Porengrößen. Kaskadenfilter haben aufgrund der großen Gesamtoberfläche schnellere Durchflusszeiten als Standardfilter, z. B. MF 10 mit den Porengrößen 200, 50, 20 und $10 \mu \mathrm{m}$ oder Microtrans mit den Porengrößen 150, 50 und $10 \mu \mathrm{m}$.

Tiefenfilter bestehen z. B. aus Dacronwolle und eliminieren Partikel aus dem Blut vornehmlich durch Adsorption als durch mechanische Trennung. Mit zunehmender Flussgeschwindigkeit werden weniger Partikel adsorbiert bzw. können sich adsorbierte Partikel wieder lösen.

\section{- Hinweise}

- ob routinemäßig Mikrofilter (10-40 $\mu \mathrm{m})$ zur Transfusion eingesetzt werden sollen, ist noch umstritten. Sie scheinen jedoch folgende Vorteile zu bieten: bei Herzchirurgie mit EKZ, Neonatologie, Massivtransfusion, Patienten mit Thrombozytopenie und bes. gefährdeten Patienten (Polytrauma, pulmonaler Vorerkrankung, Sepsis oder zu erwartender häufiger Transfusion)

- bei der Autotransfusion von präoperativ entnommenem Eigenblut scheinen Standardfilter (170-200 $\mu \mathrm{m})$ sinnvoll, wenn das entnommene Blut zur Erhaltung der Thrombozytenfunktion bis zur 6. Stunde bei Raumtemperatur auf einer Rüttelmaschine aufbewahrt wird. Bei durch Cellsaver gewonnenem Eigenblut hingegen sollten Mikrofilter (10-40 $\mu \mathrm{m})$ verwendet werden 
- ein Filterwechsel erfolgt im Allgemeinen nach 4-6 EK, spätestens wenn die Durchflussrate sinkt

\section{Komplikationen bei Transfusionen}

Die Häufigkeit von Transfusionszwischenfällen beträgt ca. 1:5000.

Man kann zwischen immunologisch und nichtimmunologisch bedingten Komplikationen unterscheiden.

\section{Hämolytische Transfusionsreaktion}

Ursache sind Antikörper gegen Erythrozyten: am häufigsten $\mathrm{AB} 0$-Unverträglichkeit, seltener bereits vor Transfusion vorhandene, hämolytisch wirksame Allo-Antikörper

- mehr als $80 \%$ sind auf menschliches Versagen, also Verwechslung von Patienten und/oder Konserven zurückzuführen

\section{Häufigkeit}

- 1:6.000-1:80.000

tödliche Reaktionen 1:250.000-1:600.000

Symptome

- Schüttelfrost und Fieber, kalter Schweiß

- Tachypnoe, Tachykardie, RR $\downarrow, \rightarrow$ Schock

- Hämolyse, Hämaturie, diffuse Blutung im Operationsgebiet

Komplikationen

DIC, akutes Nierenversagen.

\section{Therapie}

- Transfusion sofort abbrechen

- Blutentnahme für Labor, wenn möglich vor weiteren Maßnahmen:

Blutgruppenbestimmung, Kreuzprobe und AKSuchtest wiederholen. Bestimmung von Hämoglobin in Blut und Urin, Haptoglobin, Bilirubin, Kreatinin und Harnstoff, Thrombozyten, Gerinnungsstatus, Fibrinogenspaltprodukte (FSP)

- Hypotonie mit Volumengabe und ggf. Katecholaminen behandeln

- hochdosiert Kortikoide
- Diurese steigern (Volumen, Furosemid, Mannitol, Dopaminperfusor), ggf. frühzeitige Hämodialyse

- Heparinisierung bei beginnender Verbrauchskoagulopathie

- Bereitstellung von kompatiblen EK

- bei bes. schweren Reaktionen Austauschtransfusion

\section{Verzögerte hämolytische Transfusions- reaktion}

- unerklärlicher Hb-Abfall nach zunächst unauffälliger Transfusion mit mehr oder weniger ausgeprägten Hämolysezeichen

- primär niedrige Allo-Antikörpertiter beim Empfänger (negative Kreuzprobe). Derartige Reaktionen lassen sich also nicht sicher vermeiden

- nach Übertragung antigentragender Erythrozyten kommt es innerhalb weniger Tage zu einer verstärkten Antikörperbildung

\section{Nichthämolytische febrile Transfusions- reaktion (NHFT, Fieberreaktion)}

- zytotoxische Reaktion (Antigen-AntikörperReaktion) durch präformierte Antikörper des Patienten gegen Leukozyten (Thrombozyten oder Plasmaeiweiße), die mit den übertragenen Bestandteilen reagieren Häufigkeit: < 1:200 (EK), < 1:5 (TK)

- aber auch eine selten vorkommmende bakterielle Verunreinigung kommt hierfür in Betracht

\section{Posttransfusionspurpura}

- akute, isolierte Thromozytopenie mit oder ohne Blutungsneigung etwa 1 Woche nach Transfusion aufgrund der Bildung spezifischer Antikörper gegen Thrombozyten

- Inzidenz: 1:600.000 (Dtsch. Ärztebl 1999; 49: B2830), besonders Frauen >50 Jahre betroffen

- Therapie: Gabe von Immunglobulinen

\section{Allergische Reaktion}

- tritt fast ausschließlich bei Empfängern mit Hypogammaglobulinämie (IgA-Mangel) und 
Immunisierung gegen IgA-Immunoglobuline durch IgA-Übertragung auf $\Rightarrow$ Urtikaria, selten schwere Reaktionen

- kommt seit Verwendung plasmaarmer EK nur noch selten vor

\section{Transfusionsinduzierte akute Lungen- insuffizienz (TRALI-Syndrom)}

Diese Komplikation ist sehr selten und tritt fast ausschließlich durch Übertragung größerer Mengen Plasma in Form von FFP, das granulozytenspezifische Antikörper im Spenderserum enthält, auf.

\section{Graft- vs. -Host-Reaktion}

- wird bei immunsupprimierten Patienten und bei Blutsverwandten nach Übertragung von proliferationsfähigen Lymphozyten beobachtet

- durch Bestrahlung der Blutprodukte (30 Gy) zu verhindern

\section{Septischer Schock}

- Verursacht durch bakterielle Kontamination (insbesondere gramnegative Keime), meist letal endend.

\section{Infektionsübertragung}

- Übertragung von intraleukozytären Erregern (CMV, HIV, Epstein-Barr-Viren, Yersinien)

- Hepatitis B

- Hepatitis C

- Lues (Frischblut bis $72 \mathrm{~h}$ )

- Parvovirus 19 (kann bei Schwangeren (fötale Infektion), Personen mit Immundefekt oder gesteigerter Erythropoese (z.B. hämolytische Anämie) zu schweren Erkrankungen führen)

- Parasitosen insbesondere Malaria (Plasmodien), ferner Trypanosomen, Babesien, Leishmanien, Mikrofilarien und Toxoplasmen

- HTLV-II-Virus (neue Variante der CreutzfeldtJakob-Erkrankung, sicherheitshalber werden alle Spender, die sich länger als 6 Monate in

\section{Häufigkeiten unerwünschter Wirkung bei Transfusionen}

\begin{tabular}{l|l}
\hline Unerwünschte Wirkungen & Risiko je transfundierte Ein \\
\hline Hämolytische Transfusionsreaktion vom Soforttyp & \\
- ohne tödlichen Ausgang & $1: 6.000-1: 80.000$ \\
- mit tödlichem Ausgang & $1: 250.000-1: 600.000$ \\
hämolytische Transfusionsreaktion vom verzögerten Typ & $1: 1.000-1: 4.000$ \\
& $1: 100.000^{\#}$ \\
nicht-hämolytische, febrile Transfusionsreaktion (NHFT) & $<1: 200$ (EK) \\
& $<1: 5$ (TK) \\
posttransfusionelle Purpura & Einzelfälle \\
& $1: 600.000^{\#}$ \\
allergische Transfusionsreaktion & $1: 33-1: 333$ \\
- mit mildem Verlauf & $1: 20.000-1: 50.000$ \\
- mit schwerem Verlauf & $1: 5.000-1: 7.200$ \\
transfusionsassoziierte akute Lungeninsuffizienz (TRALI) & $<1: 180.000^{\#}$ \\
& $1: 400.000-1: 1.200 .000$ \\
transfusionsassoziierte Graft-Versus-Host-Krankheit (GvHD) & $1: 500.000-1: 4.700 .000$ (EK) \\
bakterielle Kontamination & $1: 900-1: 100.000$ (TK) \\
transfusionsassoziierte Virusinfektionen durch & \\
- HIV & $<1: 10^{6}$ \\
- HBV & $1: 10^{5}-1: 10^{6}$ \\
- HCV & $<1: 10^{6}$ \\
transfusionsassoziierte Parasitosen & $<1: 10^{6}$ \\
neue Variante der Creuzfeld-Jakob-Krankheit & bisher kein Fall bekannt \\
transfusionsassoziierte Gesamtmortalität (sicher/wahrscheinlich/möglich) & $1: 260.000^{\#}$ \\
& \\
\hline
\end{tabular}

Modifiziert nach Bundesärztekammer: Leitlinien zur Therapie mit Blutkomponenten und Plasmaderivaten 3. Aufl., 2003

\# Zahlen abgeleitet aus Meldungen an das britische Register Serious Hazards of Transfusion (SHOT), http://www.shotuk.org 
England aufgehalten haben von der Blutspende ausgeschlossen)

- HIV-Risiko bei FFP (Quarantänelagerung) 1:20.000.000

HIV-Risiko bei Gerinnungspräparaten (virusinaktiviert) $<$ 1:20.000.000

\section{Hypervolämie}

Tritt fast ausschließlich bei Patienten mit Herzoder Niereninsuffizienz auf.

\section{Metabolische Probleme}

Zitratintoxikation, Hyperkaliämie, Hypothermie

- bes. bei Früh- und Neugeborenen, Massivtransfusion oder ausgeprägter Leberfunktionsstörung zu beobachten

- Vermeidung durch Ca-Glukonat oder $\mathrm{CaCl}_{2}$ und vorherige Erwärmung auf $37^{\circ} \mathrm{C}$

\section{Nebenwirkungen von Leukozyten- transfusion}

- nichthämolytische, febrile Transfusionsreaktion (NHFT)

Zur Vermeidung der NHFT soll der Anteil transfundierter Leukozyten den Wert von 2,5 $\times$ $10^{8}$ pro transfundierte Einheit, der auch CALLWert („Critical Antigenic Load of Leucocytes“) genannt wird, nicht überschreiten

- Alloimmunisierung gegen HLA-Merkmale der Klasse I (notwendige gleichzeitige Übertragung von Zellen mit HLA-Antigenen der Gruppe II [BLymphos, Makrophagen, aktivierte T-Zellen])

Die für die Induktion einer Alloimmunisierung notwendige Dosis transfundierter Leukozyten wird als als CILL-Wert („Critical Immunogenetic Load of Leucocytes") bezeichnet und beträgt $5 \times 10^{6}$ pro transfundierter Einheit

- Entwicklung des Refraktärzustandes gegen Thrombozyten (inadäquater Anstieg der Thrombozytenzahlen nach Tranfusion)

- Übertragung von intraleukozytären Erregern (CMV, HIV, Epstein-Barr-Viren, Yersinien)

- Graft-vs.-Host-Reaktion

- Immunsuppression, -modulation

\section{Restleukozyten in Blutkomponenten}

\begin{tabular}{|l|c|}
\hline Blutkomponenten & Anzahl Zellen x 10 \\
\hline Vollblut & 3000 \\
Buffy-coat-haltiges EK & 3000 \\
Buffy-coat-freies EK & $400-700$ \\
Leukozyten-depletiertes EK & $<1$ \\
FFP & $<150$ \\
\hline Einzelspender-TK & $10-20$ \\
\hline Thrombozytenhochkonzentrat & $10-500$ \\
(Plasmapherese) & \\
\hline Leukozyten-depletiertes TK & $<1-50$ \\
\hline
\end{tabular}

\section{Massivtransfusion}

\section{Definitionen}

Nicht einheitlich:

- Austausch des einfachen Sollblutvolumens (70 ml/kg) innerhalb von $24 \mathrm{~h}$

- Austausch des 1,5fachen Sollblutvolumens innerhalb von $24 \mathrm{~h}$

- Austausch des halben Sollblutvolumens in $12 \mathrm{~h}$ und einer Infusionsrate von $>1,5 \mathrm{ml} / \mathrm{kg} / \mathrm{min}$

- (benötigte Transfusion $>10 \mathrm{EK}$ )

\section{Verdünnung der Gerinnungsfaktoren}

\begin{tabular}{|l|l|}
\hline $\begin{array}{l}\text { Verlust des Sollblut- } \\
\text { volumens (in \%) }\end{array}$ & $\begin{array}{l}\text { Gerinnungsfaktoren } \\
\text { (in \% der Ausgangs- } \\
\text { faktorenkonzentration) }\end{array}$ \\
\hline 50 & 60 \\
\hline 100 & 37 \\
150 & 22 \\
\hline 200 & 14 \\
\hline
\end{tabular}

(1) $\Rightarrow$ exponentieller Verlust der Gerinnungsfaktoren

\section{Auswirkungen}

\section{Körpertemperaturabfall}

- 25-30 kalte Blutkonserven $\left(4-6^{\circ} \mathrm{C}\right) \Rightarrow$ Abfall der Kerntemperatur auf $26-29{ }^{\circ} \mathrm{C}$ mit Gefahr des Kammerflimmerns

- eine Hypothermie per se löst eine Gerinnungsstörung aus

- daher Erwärmung auf $37{ }^{\circ} \mathrm{C}$, Durchlauferwärmer, Wärmegeräte 


\section{Störungen der Blutgerinnung}

- Verlustkoagulopathie durch Blutung

- Dilutionskoagulopathie durch Substitution mit kristalloiden oder kolloidalen Volumenersatzmitteln oder EK (zuerst Thrombozyten $\downarrow$ )

- Koagulopathie durch Verbrauch (Mangel an Faktor V und VIII)

Labor: PTT $\uparrow$, Quick $\downarrow$, Fibrinogen $\downarrow$, AT III $\downarrow$, Protein C $\downarrow$

- Hyperkoagulopathie (bei nur mäßiger Aktivierung der Fibrinolyse, D-Dimere)

Labor: PTT $\downarrow$

\section{Übertragung von Mikroaggregaten}

Es sollten Mikrofilter mit $10-40 \mu \mathrm{m}$ verwendet werden.

\section{Citratintoxikation bzw. Hypokalzämie}

Kalzium (ionisiertes Kalzium: Normalwert 1,1-1,4 $\mathrm{mmol} / \mathrm{l})$

- die Leber ist normalerweise in der Lage, das 10ofache der normalen Serumcitratkonzentration während einer einzelnen Passage zu metabolisieren. Bei einer Citratüberschwemmung kommt es auch zu einer Hypokalzämie, da Citrat ionisiertes Kalzium bindet

- Hypothermie, verminderte Leberdurchblutung und Hyperventilation erhöhen zusätzlich die Gefahr der Hypokalzämie

- Gesamt-Kalzium-Werte (im Labor gemessen) können irreführend sein

- deutliche Effekte auf die Gerinnung hat die ionisierte Hypokalzämie erst $<0,5 \mathrm{mmol} / \mathrm{l}$

- kardiale Phänomene können schon bei Werten $<0,75 \mathrm{mmol} / \mathrm{l} \mathrm{Ca}^{2+}$ auftreten

- $\mathrm{Ca}^{2+}$-Substitution nicht routinemäßig, sondern nurbeierniedrigtemionisiertem Kalziumspiegel, wenn keine $\mathrm{Ca}^{2+}$-Bestimmung möglich $\Rightarrow \approx 10 \mathrm{ml}$ Ca-Glukonat $10 \%$ pro 4 EK oder FFP

- $\mathrm{Ca}^{2+}$-Substitution durch Ca-Glukonat oder Ca$\mathrm{Cl}_{2}$

(I) Cave:

Ca-Glukonat und $\mathrm{CaCl}_{2}$ haben verschiedene Molarität, bei $\mathrm{CaCl}_{2}$ wird mehr ionisiertes $\mathrm{Ca}^{++}$freigesetzt (nicht an den Lebermetabolismus gebunden)

- $10 \mathrm{ml} \mathrm{Ca}$-Glukonat $10 \%(0,225 \mathrm{mmol} / \mathrm{ml})$

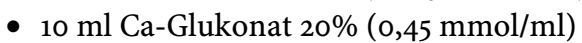

- $10 \mathrm{ml} \mathrm{CaCl}{ }_{2}$ liefert mehr ionisiertes $\mathrm{Ca}^{2+}(0,5$ $\mathrm{mmol} / \mathrm{ml}$ ) als Ca-Glukonat 10\%

\section{Hyperkaliämie}

Abhängig vom Alter der Konserven (Azidose verstärkt die Hyperkaliämie).

\section{Azidose}

Cave: Überkorrektur, da Citrat in Leber zu Bikarbonat metabolisiert wird.

\section{2,3-DPG $\downarrow$ (2,3-Diphosphoglycerin)}

- mit Linksverschiebung der $\mathrm{O}_{2}$-Bindungskurve (bei bis zu 5 Tage alten Konserven unbedeutend)

\section{Dosis}

Faustregel:

- nach Transfusion des 6.-8. EK bzw. dem 12.-14. EK $\Rightarrow$ rasche Gabe von 3-4 FFP anschl. Transfusionsverhältnis EK:FFP = 3:1

- pro 4 FFP $10 \mathrm{ml} \mathrm{Ca-Glukonat} \mathrm{10 \%} \mathrm{bzw.} 5 \mathrm{ml}$ $\mathrm{CaCl}_{2}$

- pro 10 EK 4-6 Thrombozytenkonzentrate

\section{Fremdblutsparende Maßnahmen}

\section{Präoperativ}

\section{Präoperative Eigenblutspende (EBS)}

Ind:

- planbare Operation mit zu erwartendem hohem Blutverlust ( $>1000 \mathrm{ml}$ )

KI:

- schwere respiratorische Störungen (z. B. FEV $1,5 \mathrm{l}, \mathrm{p}_{\mathrm{a}} \mathrm{O}_{2}<65 \mathrm{mmHg}$ )

- schwere kardiale Störungen (z. B. KHK mit instabiler AP, Herzinfarkt vor weniger als 6 Wochen, hochgradige Aorten-, Mitralstenose)

- Gerinnungsstörungen

- akute Infektionen (Fieber, Leukozytose)

- Anämie $(\mathrm{Hb}<11,5 \mathrm{~g} / \mathrm{dl}$ und $\mathrm{Hkt}<34 \%)$

Durchführung

- Op-Terminplanung, Beginn der EBS ca. 35-40 Tage bis max. $72 \mathrm{~h}$ vor Op 
- Entnahme von 400-500 ml Blut je Sitzung

- evtl. Substitution mit Kolloiden $(\rightarrow$ weniger kollaptische Zustände)

- Auftrennung des gewonnenen Vollblutes in EK und FFP

- primär kurze Spendeintervalle ( $<1$ Woche) $\rightarrow$ höherer Anstieg des Serumerythropoetins durch Anämisierung

- evtl. Anwendung der Bocksprungtechnik (Retransfusion älterer vorher entnommener EK bei simultaner weiterer Blutabnahme)

- Überwachung der Patienten für mind. 30-60 min

- Eisensubstitution: oral (300-900 mg Eisen-IISulfat $\approx 100-300 \mathrm{mg} \mathrm{Fe}^{2+}$ tgl) oder $100-200 \mathrm{mg}$ Eisensaccharat i.v. langsam als Kurzinfusion (Cave: allergische Reaktionen)

- evtl. Gabe von rh-Erythropoetin bei Eigenblutspende (100-150-[400] IE/kg 2mal wöchentlich s.c., ab 2. Lebensjahr) $\rightarrow$ immer simultane Eisengabe

Vorteile

- Ersatz von eigenen Gerinnungsfaktoren durch Eigen-FFP

- möglicher Infektionsschutz durch körpereigene Immunglobuline

- Stimulation der Erythropoese

\section{Präoperative Eigenplasmapherese (PPH)}

Ind:

- planbare Operation mit zu erwartenden großen Wundflächen (auch bei anämischen Patienten durchführbar)

KI:

- s. EBS, außer Anämie

Durchführung:

- Op.-Terminplanung

- Entnahme von 600-900 ml (10-15 ml/kg) Plasma je 30-90 min Sitzung

- evtl. Substitution mit Kolloiden ( $\rightarrow$ weniger kollaptische Zustände)

- Überwachung der Patienten für mind. 30-60 $\min$

- Vorteile:

- Beginn der PPH schon viele Monate vor dem Eingriff möglich
- Ersatz von eigenen Gerinnungsfaktoren

- möglicher Infektionsschutz durch körpereigene Immunglobuline

- Stimulation der Erythropoese

- auch bei sehr alten Patienten ohne Probleme durchführbar

\section{Verfahren}

- Membranfiltration

- Zentrifugation: höherer Gerinnungsfaktorengehalt und Restthrombozytenzahl als bei Membranfiltration ( $5000 \mathrm{U} / \mathrm{min} \rightarrow$ thrombozytenarmes Plasma, $3500 \mathrm{U} / \mathrm{min} \rightarrow$ thrombozytenreiches Plasma)

\section{Intra- und postoperativ}

\section{Isovolämische Hämodilution}

Ind:

- zu erwartender Blutverlust $>1000 \mathrm{ml}$ und Hkt $>34 \%$

$\mathrm{KI}$ :

- Koronar- und Herzinsuffizienz (Herzinfarkt < 3 Mo., Herzklappenfehler)

- schwere restriktive und obstruktive Lungenerkrankungen

- Anämie $<11 \mathrm{~g} / \mathrm{dl}$

- SIRS, Hypovolämie, Schock

- Fieber

- Eiweißmangel

\section{Durchführung:}

- präoperativ Entnahme von bis zu $15 \mathrm{ml} / \mathrm{kg}$ Vollblut und Ersatz durch Kolloide

Formel nach Gross:

entnehmbares BV =

geschätztes Blutvolumen $(\approx 70 \mathrm{ml} / \mathrm{kg}) \times\left(\mathrm{Hkt}_{\mathrm{o}}-\mathrm{Hkt}_{\mathrm{min}}\right)$

$$
\left(\mathrm{Hkt}_{\mathrm{o}}+\mathrm{Hkt}_{\text {min }}\right) / 2
$$

$\mathrm{Hkt}_{\mathrm{o}}=$ Ausgangshämatokrit

$\mathrm{Hkt}_{\min }=$ minimaler Hämatokrit

- Entnahme von 350-450 ml pro Beutel

- Transfusion in umgekehrter Reihenfolge der Abnahme 
- Lagerung bei Raumtemperatur auf einer Rüttelmaschine zur Erhaltung der Thrombozytenfunktion bis zur 6. h, sonst im Kühlschrank lagern

- Standardtransfusionfilter $(170-200 \mu \mathrm{m})$ verwenden

- bei der präoperativen Hämodilution kann auf einen AB0-Identitätstest vor Retransfusion verzichtet werden, wenn die Eigenblutkonserve beim Patienten verweilt und weder ein räumlicher noch personeller Wechsel zwischen Entnahme und Retransfusion erfolgt ist! Retransfusion innerhalb von 6 Stunden

Vorteile:

- Verbesserung des postoperativen Gerinnungsstatus, bessere Rheologie

- keine Schädigung der retransfundierten Erythrozyten durch den Sauger im Vergleich zur MAT

\section{Effekte}

- deutliche kardiale Nachlastsenkung: $\mathrm{EF} \uparrow, \mathrm{SV} \uparrow$, $\mathrm{HZV} \uparrow$ (über höheres SV), $\mathrm{DO}_{2} \downarrow$

- verstärkte $\mathrm{O}_{2}$-Extraktionsrate(kritischer $\mathrm{Hb}$-Wert ohne erhöhte Koronarperfusion 8,8 g/dl und mit gesteigerter Koronarperfusion bei 4,4 g/dl)

- Rechtsverschiebung der $\mathrm{O}_{2}$-Dissoziationskurve durch Zunahme von 2,3-DPG

- Abnahme der Blutviskosität

\section{Maschinelle Autotransfusion (MAT)}

Ind:

- Elektiv- oder Akut-Op mit zu erwartendem hohem Blutverlust (>1000 ml)

KI:

- Op in infektiösen oder kontaminierten Gebieten

- Tumorchirurgie

\section{Durchführung:}

- Sammeln von Blut aus dem Wundgebiet in einem sterilen Beutel (Vacufix) oder Reservoir mittels Doppellumensauger

(heparinisiertes $\mathrm{NaCl}$ läuft über ein Lumen zur Saugerspitze und wird zusammen mit dem Blut über das 2. Lumen wieder aufgesogen), Sog: 80-100 $\mathrm{mmHg}$
- Antikoagulation mit heparinisierter NaCl-Lsg. (15.000 IE Heparin auf $500 \mathrm{ml} \mathrm{NaCl} \mathrm{o,9 \%} \rightarrow$ Verhältnis zu Blut 1:5-1:10)

- die Aufbereitung (Zellseparation) des in einem Reservoir gesammelten Blutes erfolgt durch einen so genannten "Cellsaver“

- nach ausreichender Füllung des Reservoirs wird es durch eine Rollerpumpe in eine Zentrifugenglocke gepumpt. Dort wird das leichtere Plasma nach oben gedrängt und in den Abfallbeutel entleert, anschließend erfolgt ein Waschvorgang mit $\mathrm{NaCl} 0,9 \%$, der mehrfach wiederholt werden kann. Nach Beenden des Waschens wird das Erythrozytenkonzentrat in einen Transfusionsbeutel gepumpt

- ca. $80 \%$ der Erythrozyten können unzerstört zurückgewonnen werden

- Hkt der Ery-Lsg: 55-75\% (abhängig von Ausgangs-Hkt des Patienten, Verdünnung im Op.Gebiet und Anzahl der Waschvorgänge)

- hohe Qualität der Erythrozyten $\left(\mathrm{O}_{2}\right.$-Transportfunktion, Überlebenszeit und osmotische Resistenz)

- das komplette Plasma sowie Zellfragmente, freies Hämoglobin, aktivierte Gerinnungsfaktoren, aber auch Heparin werden zum größten Teil ausgewaschen

- Elimination von Medikamenten und Anästhetika

(Cave: bei Phäochromozytom nur ungenügende Auswaschung der Katecholamine)

- bei der Transfusion von durch Cellsaver gewonnenem Eigenblut sollten zur Retransfusion Mikrofilter $(10-40 \mu \mathrm{m})$ verwendet werden

- in der Regel durch Autotransfusion keine Veränderungen von Gerinnung, Elektrolytgleichgewicht und hämatologischen Werten, außer: bei hohen Autotransfusionsmengen kann es zu messbaren Veränderungen durch Heparineinschwemmung kommen (heparinisierte Waschlösung). In diesem Fall ist das Heparin durch adäquate Protamingaben $\mathrm{zu}$ antagonisieren

\section{Weitere fremdblutsparende Maßnahmen}

- gewebeschonende Operationstechnik mit akribischer Blutstillung 
- kontrollierte Hypotension (s. dort)

- Konzept der permissiven perioperativen Anämie

- postoperative Drainagenretransfusion?

- medikamentöse Beeinflussung des Blutverlustes:

- rechtzeitiges Absetzen von Thrombozytenaggregationshemmern und Umstellen auf Heparinperfusor

- Antifibrinolytika: Aprotinin (Trasylol) $\rightarrow$ Hemmung der Fibrinolyse und der durch Thrombozytenaggregationshemmer induzierten Blutungsneigung

- Desmopressin (Minirin) führt zu einer $\uparrow$ Thrombozytenausschwemmung aus Knochenmark

\section{$\mathbf{O}_{2}$-transportierende Blutersatz-} mittel (Hämoglobinmodifikationen/ Fluorocarbon-Emulsionen)

\section{Indikation für Blutersatzmittel}

- blutgruppenunabhängige Transfusion

- ggf. Erhöhung der Gewebsoxygenierung in zuvor minderversorgten Gebieten (kardial oder zerebral)

- ggf. NO-Bindung im Rahmen einer Sepsis

- ggf. zur Verbesserung der Radiosensibilität von Tumoren

- ggf. zur Reduktion eines Reperfusionsschadens

- für die einzelnen Indikationsgebiete muss ein positiver Effekt erst noch nachgewiesen werden

Bei experimentellen Versuchen mit ungereinigten, nicht modifizierten Hämoglobinlösungen trat eine Reihe von Problemen auf, die letztendlich zur Entwicklung von verschiedenen Hämoglobinmodifikationen führte.

\section{Probleme bei Einsatz von unmodifizierten Hämoglobinlösungen}

- allergische Reaktionen durch Stroma und Membranreste der Erythrozyten

- abdominelle Beschwerden, passagerer Anstieg von Leber- und Pankreasenzymen
- fragliche Nephrotoxizität (Reduktion der GFR und Tubulusobstruktion)

- kurze intravasale Verweildauer der $\mathrm{Hb}-\mathrm{Te}$ tramere, da das Tetramer in Dimere und Monomere zerfällt und dadurch renal schnell ausgeschieden wird (HWZ: 1-4 h)

- Erhöhung des kolloidosmotischen Drucks (KOD) durch Hämoglobintetramere und -dimere

- Gerinnungs- und Komplementaktivierung durch Membranfragmente der Erythrozyten

- arterielle und pulmonale Hypertonie und Bradykardie aufgrund von Vasokonstriktion durch Interaktion mit dem endothelial freigesetzten NO (HZV fällt meist $a b !)$

- erhöhte $\mathrm{O}_{2}$-Affinität (Verlust von intraerythrozytärem 2,3-DPG)

- Methämoglobinämie (Verlust der Hb-Reduktase und hierdurch vermehrte Oxydation von $\mathrm{Hb}$ zu Met-Hb)

- Beeinflussung von konventionellen photometrischen Labormessmethoden (künstliche Hämoglobinlösungen sind farbig)

- verminderter $\mathrm{CO}_{2}$-Transport (Verlust der Carboanhydrase)

- Dysphagie

- Reduktion des $\mathrm{O}_{2}$-Angebots durch Verringerung des Blutflusses (Vasokonstriktion!)

- durch verbesserte Reinigungsverfahren (Ultrapurifikation mittels Säulenchromatographie) sind zur Zeit mehrere Hämoglobinmodifikationen in klinischer Erprobung (Phase-II/III-Studien)

\section{Hämoglobinmodifikationen}

- Verlängerung der intravasalen Verweildauer von Blutersatzmitteln auf 16-36 h und Normalisierung der erhöhten KOD durch (• Abb. 61.1):

- intermolekulares Cross-linking

- Polymerisation von einzelnen Hb-Molekülen zu Polymeren, z. B. durch Glutaraldehyd

- Konjugation an Makromoleküle, z. B. Polyethylenglykol, Polyvinylpyrrolidon, Dextrane

- Liposomenverkapselung $(\varnothing<1 \mu \mathrm{m})$

- Verringerung der $\mathrm{O}_{2}$-Affinität

- erhöhte $\mathrm{O}_{2}$-Affinität durch 2,3-Diphosphoglycerinverlust $\rightarrow$ Gabe von Pyridoxal-5-phosphat reduziert $\mathbf{O}_{2}$-Affinität und wirkt diesem 


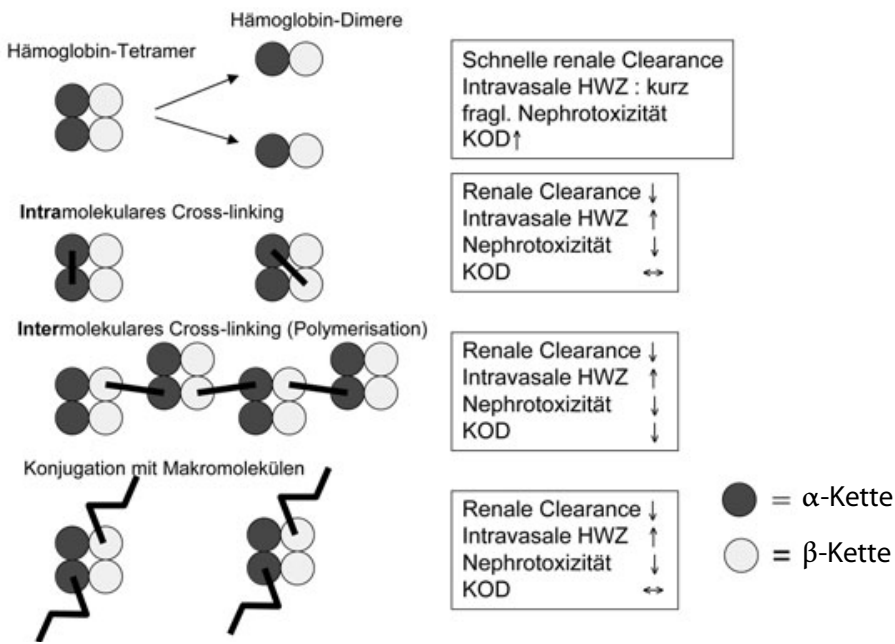

- Abb. 61.1. Hämoglobinmodifikationen (mod. nach Standl, AINS 1998, 33:701)
Effekt entgegen $\left(\mathbf{P}_{50}\right.$ ist der $\mathrm{O}_{2}$-Partialdruck, bei dem $50 \%$ des Hämoglobins gesättigt sind. Unter physiologischen Konditionen beträgt $\mathrm{P}_{50}=26-28 \mathrm{mmHg}$, bei 2,3-DPG-Verlust nur noch 12-15 mmHg) $\rightarrow$ durch Pyridoxilierung $\operatorname{der} \beta$-Untereinheit mit Pyridoxal-5-Phosphat, 2-Nor-2-Formylpyridoxal-5-Phosphat, DiPyridoxal-Tetraphosphat $\rightarrow \mathrm{P}_{50}$ ansteigend von 12 auf $24 \mathrm{mmHg}$

- Verringerung der Nephrotoxizität - durch Abbau von modifizierten Hb-Lösungen im retikuloendothelialen System (RES)

\section{Polymerisiertes ultragereinigtes Rinderhämoglobin (Hempure/USA)}

- durch Glutaraldehyd polymerisiertes ultragereinigtes Rinderhämoglobin, dessen reduzierte $\mathrm{O}_{2}$-Affinität nicht über 2,3-DPG, sondern über Chloridionen kontrolliert wird ( $\mathrm{Hb}: 13 \mathrm{mg} / \mathrm{dl}$ und KOD: $15 \mathrm{mmHg}$ )

- die desoxygenierte Form ist bei Raumtemperatur ca. 2 Jahre lagerungsfähig!

- Nebenwirkungen: Anstieg des arteriellen Mitteldruckes um max. $18 \%$, des SVR um bis ca. $40 \%$ $\rightarrow$ bedingt durch Interaktionen der Hämoglobinmoleküle mit dem NO-Stoffwechsel (Unterschiede zwischen Oxy- und Desoxyhämoglobin) infolge der Wirkung von Resten der Erythrozytenmembranen in Form von Phospholipiden (bei anderen nicht hochgereinigten Präparaten nachgewiesen!) $\rightarrow$ pulmonale und systemische Vasokonstriktion und konsekutiver Abfall des HZV und vermehrter peripherer Sauerstoffextraktion $\left(\mathrm{PvO}_{2} \downarrow\right) \rightarrow$ reaktive Vasokonstriktion als Antwort auf eine Hyperoxygenierung des Gewebes unter Rinderhämoglobin

- intravasale HWZ: ca. 8,5 h

- Metabolisierung: keine renale Ausscheidung, sondern Speicherung von größeren Molekülen im RES $\rightarrow$ fraglicher immunsuppressiver Effekt

- veränderte $\mathrm{O}_{2}$-Sättigungskurve bei Rinderhämoglobin $\left(\mathrm{p}_{\mathrm{a}} \mathrm{O}_{2}\right.$ von $100 \mathrm{mmHg}=80 \%, \mathrm{p}_{\mathrm{a}} \mathrm{O}_{2}$ von 140-150 $\mathrm{mmHg}=$ infolge des flachen oberen Kurvenverlaufs nur ca. $85 \%$ )

- Dosierung: ca. 0,4 g/kg

- bis jetzt sind keine toxischen Nebenwirkungen bezüglich Blutgerinnung, Leber- und Nierenfunktion in den Phase III-Studien aufgetreten!

\section{Diaspirin-vernetztes Hämoglobin} (HemAssist/USA)

- Diaspirin ist eine vernetzte Hämoglobin-Lösung (DCLHb) der Firma Baxter

- intramolekulare Vernetzung der $\alpha$-Untereinheiten von menschlichem, hitzeinaktiviertem Hämoglobin mit Diaspirin $\rightarrow$ Stabilität und ähnliche $\mathrm{O}_{2}$-Bindungskurve wie natürliches Hämoglobin $\rightarrow$ Verbesserung der Mikrozirkulation (tierexperimentell)

- Lagerung: tiefgefroren oder für $48 \mathrm{~h}$ gekühlt 
Überblick über die zur Zeit im klinischen Einsatz getesteten Hämoglobinpräparate

\begin{tabular}{|c|c|c|}
\hline Provenienz & Modifikation & Name/Hersteller \\
\hline Human & Intramolekulares $\alpha$ - $\alpha$-cross-linking mit Diaspirin (DCLHB) & HemAssist (Baxter, USA) \\
\hline Human & Glutaraldehyd-Polymerisation & PolyHeme (Northfield, USA) \\
\hline Human & Intra- und intermolekulares Cross-linking mit O-Raffinose & Hemolink (Hemosol, Kanada) \\
\hline Bovin & Polyethylenglykol-Konjugation & PEG-Hb (Enzon, USA) \\
\hline $\begin{array}{l}\text { Bovin } \\
\text { Von E. coli } \\
\text { produziertes, } \\
\text { rekombinantes }\end{array}$ & $\begin{array}{l}\text { Glutaraldehyd-Polymerisation } \\
\alpha \text {-Kettenfusion } \\
\beta \text {-Kettenmutation }\end{array}$ & $\begin{array}{l}\text { Hempure (Biopur, USA) } \\
\text { Optro (Samatogen, USA) }\end{array}$ \\
\hline
\end{tabular}

- HWZ: bis $36 \mathrm{~h}$ (tierexperimentell)

- $\mathrm{P}_{50}=32 \mathrm{mmHg}$

- Nebenwirkungen:

- Anstieg des MAP (Mechanismus noch nicht aufgeklärt: Sensibilisierung von Endothelinrezeptoren, Freisetzung von Endothelin, NO-Bindung oder Modulation von Adrenorezeptoren) $\rightarrow$ bei repetitiver Gabe kommt es zu einem geringeren Blutdruckanstieg (Tachyphylaxie)

- Hyperbilirubinämie und Ikterus

Phase-III-Studie wurde aufgrund eines schlechteren Ergebnisses bei hämorrhagischen Patienten eingestellt!

Bis heute unklar sind folgende Punkte:

- Inzidenz von allergischen Reaktionen (Verunreinigung mit Erythrozytenstroma bzw. Bildung von Antikörpern der IgG-Klasse)

- renale Toxizität infolge Tubulusverstopfung mit Hämoglobinmonomeren

- Interaktion mit endogenem NO und anderen vasotonussteuernden Systemen (Freisetzung von Endothelin, Sensibilisierung von peripheren $\alpha$ Rezeptoren)

\section{Fluorocarbon-Emulsionen}

- wasserunlösliche Kohlenstoff-Fluor-Verbindungen, welche in eine Lecithin-Emulsion eingebracht werden (Cave: Patienten mit Ei-Allergie!) $\rightarrow$ Erzeugung von verträglichen Tröpfchen bestimmter Größe

- Abbau erfolgt mit der Aufnahme der Tröpfchen in das retikuloendotheliale System (RES) und
Abbau über Tage zu einzelnen Fluorocarbonmolekülen, welche nach Abgabe ans Blut und Bindung an Blutfette zur Lunge transportiert und dort exhaliert werden $\rightarrow$ kein Metabolismus der Fluorocarbone

NW:

- grippeähnliche Symptome mit Myalgien und Fieber, Erhöhung der Serumamylase und Pankreatitiden, passagerer Thrombozytenabfall um ca. $10-20 \%$ vom Ausgangswert zwischen dem 3. und 7. Tag nach Applikation, geringere Transportkapazität für $\mathrm{O}_{2}$ im Vergleich zu Hämoglobinlösungen

\section{Transfusionsgesetz}

Blut und Blutkomponenten unterliegen in Deutschland dem Arzneimittelgesetz

\section{Wesentliche Punkte des Transfusionsgesetzes}

- Inkrafttreten am 07.07.1998 mit Ausnahme von $\$ 15$ TFG (Qualitätssicherung) und \$ 22 TFG (epidemiologische Daten). Inkrafttreten von $\$ 15$ am 07.07.2001, $\$ 22$ am 07.07.2000

- Dokumentationspflicht gemäß $\$ 14$ für folgende Produkte:

- Eigenblut

- Fremdblut + Komponenten (Erythrozytenkonzentrate etc.) 
- Blutprodukte bzw. Plasmaderivate ( $\alpha_{1}$-Proteinaseninhibitoren, [Albumin], C1-Inhibitor, Fibrinkleber, Fibrinogen, Gerinnungsfaktoren VII, VIII, IX, XIII; Prothrombinkomplex-Präparate (PPSB), AT III, FFP, Immunglobuline, Interferone, Plasminogen, Plasmaproteinlösung, Protein C, Serumcholinesterase, Transferinfaktor, G-CSF im Rahmen der Stammzelltransfusion [sonst nicht dokumentationspflichtig])

\section{- Stammzellen}

- Dokumentation auch von nicht angewandten/ applizierten Blutprodukten $(\$ 17)$

- Überwachung der Einhaltung des TFG durch einen zu benennenden Transfusionsverantwortlichen bzw. Bildung einer Transfusionskommission in Krankenhäusern mit Spendeeinrichtung oder Transfusionsmedizinischem Institut

- Meldung des jährlichen Hämostatikaverbrauchs bzw. Anzahl der behandelten Patienten mit angeborener Hämostasestörung ( $\$$ 21)

- Meldung von unerwünschten Arneimittelnebenwirkungen (UAW) gemäß $\$ 16$
- Implemetierung einer Qualitätssicherung (\$15)

- bei V.a. transfusionsbedingte Infektionen Unterrichtungspflicht der betroffenen Spendeeinrichtung bzw. des pharmazeutischen Unternehmens und ggf. Rückverfolgung der spendenden Personen (\$19)

- Aufbewahrung der Dokumentation für 15 Jahre

- weitere Informationen, Links und Orginaltexte finden sich u. a. an folgenden Stellen:

- http://www.bundesanzeiger.de (Orginaltext des Transfusionsgesetzes)

- http://www.pei.de(Paul-Ehrlich-Institut:Transfusionsgesetz mit Kommentar und Diskussion)

- http://www.rki.de (Voten des Arbeitskreis Blut)

- http://www.aerzteblatt.de (offizielle Verlautbarungen der Bundesärztekammer)

- http://www.dgti.de (Gesellschaft für Transfusionsmedizin und Immunhämatologie)

- http://www.gth.de (Gesellschaft für Thrombose und Hämostaseforschung) 